\title{
Efficient evaluation of antenna fields by a time-domain multipole analysis
}

\author{
J. Adam and L. Klinkenbusch \\ Institute for Electrical and Information Engineering, Christian-Albrechts-Universität zu Kiel, Germany
}

\begin{abstract}
The contribution describes a systematic method to efficiently determine frequency-domain electromagnetic antenna fields and characteristics for a broad spectrum via a single time-domain (e.g., Finite-Difference Time-Domain, FDTD) calculation. From a time-domain simulation of an antenna driven by a wide-band signal, a single modified Fourier transformation yields the frequency-domain multipole amplitudes. The corresponding multipole expansions are valid for the entire spectrum of the input pulse and at any point outside a minimum sphere enclosing the antenna. This allows a computationally cheap and elegant post-processing of arbitrary antenna characteristics. As an example of use the method is applied to determine high-resolution threedimensional radiation patterns of an antipodal Vivaldi antenna.
\end{abstract}

\section{Introduction}

Multipole techniques have often been employed in the context of antenna fields. For example, they have been used to derive general properties of linear antennas such as the relations between the field modal amplitudes and the elements of the antenna's equivalent circuit (Chu, 1948), and to derive expressions for the antenna $Q$ and the bandwidth (Fante, 1969; Yaghjian and Best, 2005). Moreover, multipole expansions in spherical, in cylindrical, and in Cartesian coordinates are the basis to calculate the probe-corrected far-field from measured or estimated values of the antenna near field (Hansen, 1988). The present method provides a receipt to obtain the frequency-domain spherical-multipole amplitudes from given time-domain near-field data. According to the surface equivalence theorem (Balanis, 1989) the electric farfield is completely determined by the tangential components of the field data (and resulting surface currents) on a closed

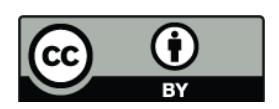

Correspondence to: J. Adam (ja@tf.uni-kiel.de) surface, surrounding all scattering objects. Here, a discrete version of this theorem, namely the spherical-multipole interface (Klinkenbusch, 1995), is applied: In parallel to the time-stepping process of a time-domain near-field solver the tangential near-field on an arbitrarily chosen Huygens surface (that is, an imaginary closed surface enclosing the antenna structure) is replaced by a suitably chosen number of equivalent electric and magnetic elementary dipoles. The dipoles' amplitudes are temporally linearly interpolated. The corresponding time-domain spherical-multipole amplitudes are then deduced by means of the inverse Fourier transform of the bilinear form of the free-space dyadic Green's function in spherical coordinates, valid at far-field observation points.

Conventional time-domain near-field solvers (like FDTD) have intrinsic near-to-far-field routines that are based on the method of retarded potentials (Luebbers et al., 1991) and therefore make use of the closed form of this Green's function. As a consequence, a new integration over the near-field values is necessary for each observation point, and/or suitable interpolation routines are necessary (Boag and Shlivinski , 2007), which might be ineffective for large numbers of desired far-field points. In contrast, the time-domain multipole amplitudes have to be calculated only once, and the field values in arbitrary observation points are obtained by a simple superposition. Moreover, in the case of a wideband transient pulse as the antenna signal, a numerically performed modified Fourier transform of the time-domain multipole amplitudes directly yields the frequency-domain multipole amplitudes of the antenna field for the entire frequency spectrum of this transient pulse. These frequency-domain multipole amplitudes can be used to analyze the antenna field (and other quantities of interest) at any point outside a minimum sphere enclosing all radiating elements (not just in the far-field) and at all frequencies of interest.

For the description of the present method, we first give a brief introduction to spherical-multipole expansions and a short description of the spherical-multipole interface. After that, we show how to efficiently implement the necessary convolution integrals into the time-stepping process of the

Published by Copernicus Publications on behalf of the URSI Landesausschuss in der Bundesrepublik Deutschland e.V. 
near-field solver. Finally we deduce the frequency-domain multipole amplitudes directly from their time-domain counterparts. To validate the present method and to demonstrate its capability of treating radiation problems of practical interest, the implementation of the method into a standard FDTD solver is applied to determine high resolution three dimensional radiation patterns of an ultra wide-band balanced antipodal Vivaldi antenna.

\section{Frequency- and time-domain spherical multipole field representations}

In any homogenous domain the frequency-domain electromagnetic field at circle frequency $\omega$ can be represented in spherical coordinates $(r, \vartheta, \varphi)$ by the spherical-multipole expansion

$$
\begin{aligned}
& \boldsymbol{E}(\boldsymbol{r}, \omega)= \\
& =\sum_{n, m}\left\{A_{n, m}(\omega) \boldsymbol{N}_{n, m}(\boldsymbol{r})+\frac{Z}{j} B_{n, m}(\omega) \boldsymbol{M}_{n, m}(\boldsymbol{r})\right\}, \\
& \boldsymbol{H}(\boldsymbol{r}, \omega)= \\
& =-\sum_{n, m}\left\{\frac{j}{Z} A_{n, m}(\omega) \boldsymbol{M}_{n, m}(\boldsymbol{r})+B_{n, m}(\omega) \boldsymbol{N}_{n, m}(\boldsymbol{r})\right\} .
\end{aligned}
$$

Here, $Z=\sqrt{\mu / \epsilon}$ denotes the intrinsic impedance of the surrounding media, and we have used the abbreviation

$\sum_{n, m}=\sum_{n=1}^{\infty} \sum_{m=-n}^{n}$

The coefficients of these expansions are referred to as the (frequency-domain) electric and magnetic multipole amplitudes $A_{n, m}(\omega)$ and $B_{n, m}(\omega)$, respectively. The vector spherical-multipole functions are deduced from particular solutions of the Helmholtz equation in spherical coordinates as

$$
\begin{aligned}
\boldsymbol{M}_{n, m}(\boldsymbol{r})= & z_{n}(\kappa r) \boldsymbol{m}_{n, m}(\vartheta, \varphi), \\
\boldsymbol{N}_{n, m}(\boldsymbol{r})=- & \frac{z_{n}(\kappa r)}{\kappa r} n(n+1) Y_{n, m}(\vartheta, \varphi) \hat{\boldsymbol{r}} \\
& \quad-\frac{1}{\kappa r} \frac{d}{d r}\left[r z_{n}(\kappa r)\right] \boldsymbol{n}_{n, m}(\vartheta, \varphi) .
\end{aligned}
$$

Here, the angular-dependent factors consist of the vector functions

$$
\begin{aligned}
\boldsymbol{m}_{n, m}(\vartheta, \varphi) & =-\frac{1}{\sin (\vartheta)} \frac{\partial Y_{n, m}(\vartheta, \varphi)}{\partial \varphi} \hat{\boldsymbol{\vartheta}}+\frac{\partial Y_{n, m}(\vartheta, \varphi)}{\partial \vartheta} \hat{\boldsymbol{\varphi}} \\
\boldsymbol{n}_{n, m}(\vartheta, \varphi) & =\frac{\partial Y_{n, m}(\vartheta, \varphi)}{\partial \vartheta} \hat{\boldsymbol{\vartheta}}+\frac{1}{\sin (\vartheta)} \frac{\partial Y_{n, m}(\vartheta, \varphi)}{\partial \varphi} \hat{\boldsymbol{\varphi}}
\end{aligned}
$$

and are based on the surface spherical-harmonics

$$
Y_{n, m}(\vartheta, \varphi)=\sqrt{\frac{2 n+1}{4 \pi} \frac{(n-m) !}{(n+m) !}} P_{n}^{m}(\cos \vartheta) e^{j m \varphi} .
$$

They particularly form a complete set of orthogonal vector functions on the $\mathbb{R}^{3}$-sphere. Here, $P_{n}^{m}$ denotes an associated Legendre function of the first kind, see (Abramowitz and Stegun, 1972) for details. Moreover, $z_{n}(\kappa r)$ represents a spherical Bessel function, where $\kappa=\omega \sqrt{\mu \varepsilon}$ denotes the wavenumber. In particular, spherical Bessel function of the first kind $z_{n}=j_{n}$ are chosen to match regularity at the origin, whereas spherical Hankel functions of the 2nd kind $z_{n}=h_{n}^{(2)}$ are chosen to coincide with Sommerfeld's radiation condition.

With a suitable approximation of the mentioned spherical Hankel function, the electric far-field can be written as

$$
\begin{array}{r}
\boldsymbol{E}_{\infty}(\boldsymbol{r}, \omega)=-\frac{e^{-j \kappa r}}{\kappa r} \sum_{n, m} j^{n}\left\{A_{n, m}(\omega) \boldsymbol{n}_{n, m}(\vartheta, \varphi)\right. \\
\left.-Z_{0} B_{n, m}(\omega) \boldsymbol{m}_{n, m}(\vartheta, \varphi)\right\} .
\end{array}
$$

This field is easily Fourier transformed to the time-domain electric far-field representation (Oetting and Klinkenbusch, 2005):

$$
\begin{array}{r}
\boldsymbol{e}_{\infty}(\boldsymbol{r}, t)=-\frac{1}{r} \sum_{n, m}\left\{a_{n, m}\left(t-\frac{r}{v_{c}}\right) \boldsymbol{n}_{n, m}(\vartheta, \varphi)\right. \\
\left.-Z_{0} b_{n, m}\left(t-\frac{r}{v_{c}}\right) \boldsymbol{m}_{n, m}(\vartheta, \varphi)\right\},
\end{array}
$$

where $v_{c}=1 / \sqrt{\mu_{0} \epsilon_{0}}$ denotes the velocity of light. The timedomain multipole amplitudes $a_{n, m}$ and $b_{n, m}$ are formally related to their frequency-domain counterparts $A_{n, m}$ and $B_{n, m}$ by the (modified) Fourier transforms

$$
\begin{aligned}
& a_{n, m}(t)=j^{n} \frac{v_{c}}{2 \pi} \int_{-\infty}^{\infty} \frac{A_{n, m}(\omega)}{\omega} e^{+j \omega t} d \omega, \\
& b_{n, m}(t)=j^{n} \frac{v_{c}}{2 \pi} \int_{-\infty}^{\infty} \frac{B_{n, m}(\omega)}{\omega} e^{+j \omega t} d \omega .
\end{aligned}
$$

\section{Time-domain near-to-far-field transformation by a spherical-multipole interface}

In order to determine the time-domain multipole amplitudes $a_{n, m}(t)$ and $b_{n, m}(t)$, the spherical-multipole interface (Klinkenbusch, 1995) is employed: The field components on a surface $S$, surrounding all radiating sources (Huygenssurface), are replaced by a finite number of appropriately chosen electric and magnetic elementary dipoles. Assuming $N_{e l}$ electric dipoles at locations $\boldsymbol{r}_{e l}^{[i]}, i \in\left\{1, \ldots, N_{e l}\right\}$ and $N_{\text {mag }}$ magnetic dipoles at $\boldsymbol{r}_{\text {mag }}^{[i]}, i \in\left\{1, \ldots, N_{\text {mag }}\right\}$, the corresponding current moments $\boldsymbol{c}_{e l}^{[i]}$ and $\boldsymbol{c}_{\text {mag }}^{[i]}$ are obtained by the near-field data as

$$
\begin{gathered}
\boldsymbol{c}_{e l}\left(\boldsymbol{r}^{[i]}\right)=\hat{\boldsymbol{n}}_{S}^{[i]} \times \boldsymbol{H}\left(\boldsymbol{r}^{[i]}\right) \Delta_{f}^{[i]}, \\
\boldsymbol{c}_{\mathrm{mag}}\left(\boldsymbol{r}^{[i]}\right)=-\hat{\boldsymbol{n}}_{S}^{[i]} \times \boldsymbol{E}\left(\boldsymbol{r}^{[i]}\right) \Delta_{f}^{[i]} .
\end{gathered}
$$


$\Delta_{f}^{[i]}$ denotes the corresponding surface area of the particular surface element, while $\hat{\boldsymbol{n}}_{S}^{[i]}$ represents the outwardly oriented unit vector perpendicular to that surface element. Now the time-domain multipole amplitudes are easily found by a summation of all of the dipoles fields via suitable convolution integrals containing the current moments $c_{e l}^{[i]}$ and $\boldsymbol{c}_{\text {mag }}^{[i]}$ (Oetting and Klinkenbusch, 2005; Klinkenbusch and Oetting, 2007). In a discrete form (at time-steps $\left.k \Delta_{t}(k=0,1,2, \ldots)\right)$ and applying a temporal linear interpolation of the discrete current moments $\boldsymbol{c}_{e l}^{k[i]}:=\boldsymbol{c}_{e l}^{[i]}\left(k \Delta_{t}\right)$ and $\boldsymbol{c}_{\text {mag }}^{k[i]}:=\boldsymbol{c}_{\text {mag }}^{[i]}\left(k \Delta_{t}\right)$, we arrive at the following numerically implementable representations:

$$
\begin{array}{r}
a_{n, m}\left(k \Delta_{t}\right)=\frac{(-1)^{n+m}}{2 n(n+1)} \times \\
\times\left\{Z \sum _ { i = 1 } ^ { N _ { e l } } \sum _ { l = - w } ^ { w - 1 } \left[\psi_{n}^{l,[i]}\left(c_{e l}^{k-l[i]}+l \Delta_{c e}^{k-l}\right) \cdot \zeta_{n, m}^{[i]}\right.\right. \\
\left.-\frac{\chi_{n}^{l,[i]}}{\Delta_{t}} \boldsymbol{\Delta}_{c e}^{k-l} \cdot \boldsymbol{\xi}_{n, m}^{[i]}\right] \\
\left.+\sum_{i=1}^{N_{\operatorname{mag}}} \sum_{l=-w}^{w-1} \frac{\psi_{n}^{l,[i]}}{\Delta_{t}} \boldsymbol{\Delta}_{c m}^{k-l} \cdot \boldsymbol{\pi}_{n, m}^{[i]}\right\},
\end{array}
$$

and

$$
\begin{array}{r}
b_{n, m}\left(k \Delta_{t}\right)=\frac{(-1)^{n+m}}{2 n(n+1)} \times \\
\times\left\{\frac { 1 } { Z } \sum _ { i = 1 } ^ { N _ { \mathrm { mag } } } \sum _ { l = - w } ^ { w - 1 } \left[\psi_{n}^{l,[i]}\left(\boldsymbol{c}_{\mathrm{mag}}^{k-l[i]}+l \boldsymbol{\Delta}_{c m}^{k-l}\right) \cdot \boldsymbol{\zeta}_{n, m}^{[i]}\right.\right. \\
\left.-\frac{\chi_{n}^{l,[i]}}{\Delta_{t}} \boldsymbol{\Delta}_{c m}^{k-l} \cdot \boldsymbol{\xi}_{n, m}^{[i]}\right] \\
\left.-\sum_{i=1}^{N_{e l}} \sum_{l=-w}^{w-1} \frac{\psi_{n}^{l,[i]}}{\Delta_{t}} \boldsymbol{\Delta}_{c e}^{k-l} \cdot \boldsymbol{\pi}_{n, m}^{[i]}\right\} .
\end{array}
$$

Here we have used the abbreviations

$$
\Delta_{c e}^{l}=\left(c_{e l}^{l,[i]}-c_{e l}^{l-1,[i]}\right)
$$

and $\quad \Delta_{c m}^{l}=\left(c_{\mathrm{mag}}^{l,[i]}-\boldsymbol{c}_{\mathrm{mag}}^{l-1,[i]}\right)$

for all $l \in \mathbb{Z}$, where the three vector functions $\zeta_{n, m}^{[i]}, \boldsymbol{\xi}_{n, m}^{[i]}$ and $\boldsymbol{\pi}_{n, m}^{[i]}$ are defined by

$$
\begin{aligned}
\zeta_{n, m}^{[i]} & =\frac{1}{r^{[i]}}\left(n(n+1) Y_{n,-m}^{[i]} \hat{r}+\boldsymbol{n}_{n,-m}^{[i]}\right), \\
\boldsymbol{\xi}_{n, m}^{[i]} & =\frac{1}{v_{c}}\left(n(n+1) Y_{n,-m}^{[i]} \hat{r}+2 \boldsymbol{n}_{n,-m}^{[i]}\right), \\
\boldsymbol{\pi}_{n, m}^{[i]} & =\frac{1}{v_{c}} \boldsymbol{m}_{n,-m}^{[i]} .
\end{aligned}
$$

$$
\begin{array}{lcl}
\text { Note } & \text { that } & Y_{n,-m}^{[i]}=Y_{n,-m}\left(\vartheta^{[i]}, \varphi^{[i]}\right), \\
\boldsymbol{m}_{n,-m}^{[i]}=\boldsymbol{m}_{n,-m}\left(\vartheta^{[i]}, \varphi^{[i]}\right)
\end{array} \quad \text { and } \quad \begin{aligned}
& \boldsymbol{n}_{n,-m}^{[i]}=\boldsymbol{n}_{n,-m}\left(\vartheta^{[i]}, \varphi^{[i]}\right) .
\end{aligned}
$$

The two scalar factors $\chi_{n}^{l,[i]}$ and $\psi_{n}^{l,[i]}$ represent integrals within the interval $\left[l \frac{\Delta_{t} v_{c}}{r^{[i]}},(l+1) \frac{\Delta_{t} v_{c}}{r^{[i]}}\right],(l \in \mathbb{Z})$, which involve Legendre polynomials and which can be evaluated completely analytically. The first factor reads

$\psi_{n}^{l,[i]}=\frac{\tau_{l+1}^{[i]} P_{n}^{\prime}\left(t_{l+1}^{[i]}\right) \operatorname{rect}\left(t_{l+1}^{[i]}\right)-\tau_{l}^{[i]} P_{n}^{\prime}\left(t_{l}^{[i]}\right) \operatorname{rect}\left(t_{l}^{[i]}\right)}{n(n+1)}$,

where $P_{n}$ denotes a Legendre polynomial of the first kind and order $n, t_{l}^{[i]}=l \frac{\Delta_{t} v_{c}}{r^{[i]}}$, and $\tau_{l}^{[i]}=\left(t_{l}^{[i]^{2}}-1\right)$. The rectangle function is defined in the usual way as

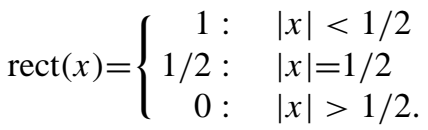

In the case $n>1$ the second factor reads

$\chi_{n}^{l,[i]}=\frac{\lambda_{n}^{l+1,[i]} \operatorname{rect}\left(t_{l+1}^{[i]}\right)-\lambda_{n}^{l,[i]} \operatorname{rect}\left(t_{l}^{[i]}\right)}{(n-1)(n+2)}$,

with

$\lambda_{n}^{l,[i]}=\tau_{l}^{[i]}\left(t_{l}^{[i]} P_{n}^{\prime}\left(t_{l}^{[i]}\right)-P_{n}\left(t_{l}^{[i]}\right)\right)$.

In the case $n=1$ we finally derive

$$
\begin{array}{r}
\chi_{1}^{l,[i]}=\frac{1}{3}\left[t_{l+1}^{[i]}{ }^{3} \operatorname{rect}\left(t_{l+1}^{[i]}\right)-t_{l}^{[i]}{ }^{3} \operatorname{rect}\left(t_{l}^{[i]}\right)\right. \\
\left.+\left(\operatorname{rect}\left(t_{l+1}^{[i]}\right)-\operatorname{rect}\left(t_{l}^{[i]}\right)\right)^{2}\right] .
\end{array}
$$

In order to efficiently fit the calculation of this expressions into the time-stepping of the time-domain near-field solver, the Legendre polynomials can be calculated by a recently proposed time-recursive scheme (Adam and Klinkenbusch, 2006):

$$
\left(\begin{array}{c}
P_{n}\left(t_{l+1}^{[i]}\right) \\
\tau_{l+1}^{[i]} P_{n}^{\prime}\left(t_{l+1}^{[i]}\right)
\end{array}\right)=\mathbf{M}_{l}^{[i]}\left(\begin{array}{c}
P_{n}\left(t_{l}^{[i]}\right) \\
\tau_{l}^{[i]} P_{n}^{\prime}\left(t_{l}^{[i]}\right)
\end{array}\right)
$$

where the update operator $\mathbf{M}_{l}^{[i]}$ is given by

$$
\begin{aligned}
\mathbf{M}_{l}^{[i]} & =\frac{4 \tau_{l+1}^{[i]}}{4 \tau_{l+1}^{[i]}-\Delta^{[i]^{2}} n(n+1)} \times \\
& \times\left(\begin{array}{cc}
\frac{4 \tau_{l+1}^{[i]}+\Delta^{[i]^{2}} n(n+1)}{4 \tau_{l+1}^{[i]}} & \frac{\Delta^{[i]} \tau_{l+1}^{[i]}+\Delta^{[i]} \tau_{l}^{[i]}}{2 \tau_{l}^{[i]} \tau_{l+1}^{[i]}} \\
\Delta^{[i]} n(n+1) & \frac{4 \tau_{l}^{[i]}+\Delta^{[i]^{2}} n(n+1)}{4 \tau_{l}^{[i]}}
\end{array}\right),
\end{aligned}
$$

with temporal increment $\Delta^{[i]}=\frac{\Delta_{t} v_{c}}{r^{[i]}}$.

\section{Evaluation of antenna far- and near-fields}

Consider an arbitrary linear antenna which radiates into the free unbounded space. The related electromagnetic problem is solved by a suitable near-field solver (as FDTD) using a 


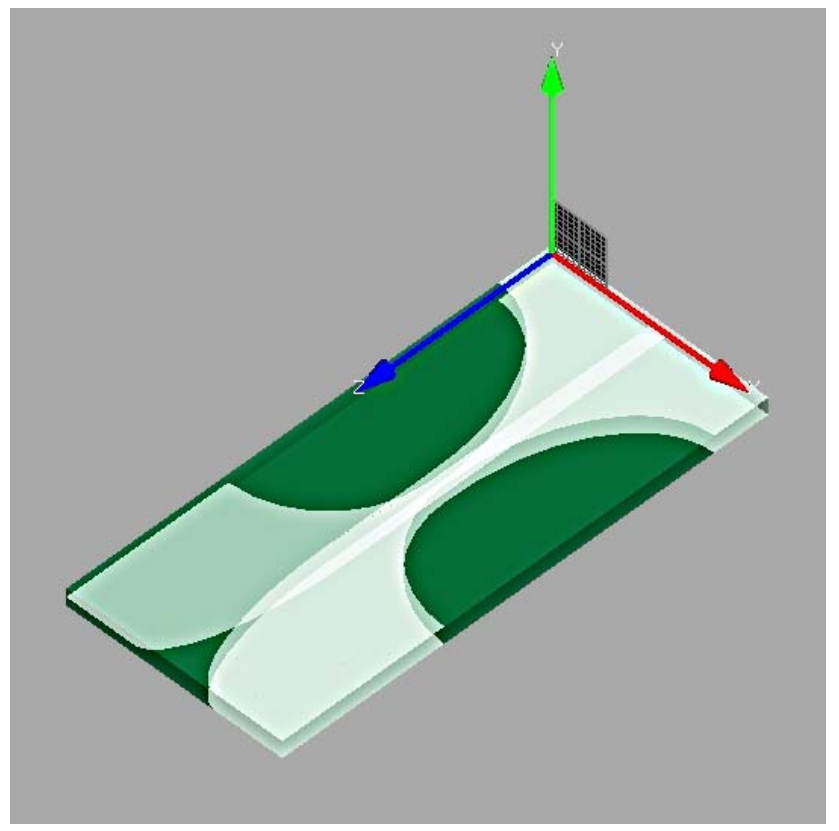

Fig. 1. CAD drawing of the antipodal Vivaldi antenna.

wide-band pulse as the antenna input signal. The corresponding time-domain far-field multipole amplitudes are then evaluated as proposed in Sect. 3. The corresponding frequencydomain multipole amplitudes $A_{n, m}$ and $B_{n, m}$ are formally related to the time-domain ones by the modified Fourier transforms

$$
\begin{aligned}
& A_{n, m}(\omega)=j^{-n} \frac{\omega}{v_{c}} \int_{-\infty}^{\infty} a_{n, m}(t) e^{-j \omega t} d t, \\
& B_{n, m}(\omega)=j^{-n} \frac{\omega}{v_{c}} \int_{-\infty}^{\infty} b_{n, m}(t) e^{-j \omega t} d t .
\end{aligned}
$$

Thus, we obtain the frequency-domain multipole amplitudes for the entire range of the spectrum of the input pulse by a (generally numerically performed but cheap) Fourier transform. The frequency-domain electromagnetic field at any desired frequency and any desired point outside the minimum sphere is then analytically obtained by means of simply evaluating (1) and (2).

As a consequence, these representations can be used to conveniently and analytically post-process the antenna nearand far-field and to obtain any related parameters.

\section{Numerical Results: Vivaldi Antenna Fields by means of an FDTD solver}

The proposed multipole-based near-to-far-field algorithm has been integrated into a (for research purposes provided) source-code of XFdtd, a commercially available 3-D

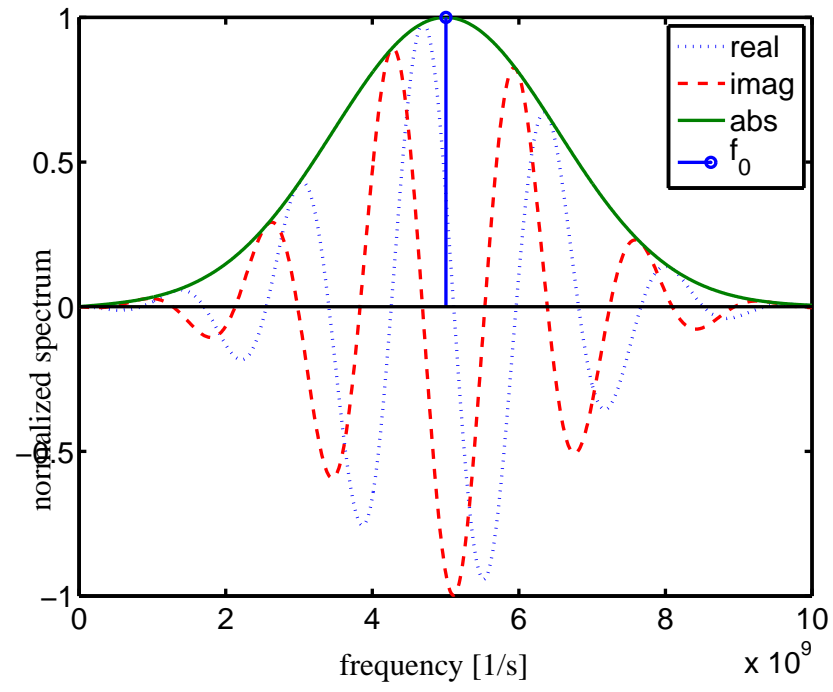

Fig. 2. Spectrum of the transient feed pulse, defined by $\Delta_{t}=2.9345 \mathrm{ps}, f_{c}=5 \mathrm{Ghz}$, and $\beta=200$ time-steps.

FDTD code (Remcom Inc., 1998) (scattered field formulation, PML). In this case, the near-field data is given in discrete form (Taflove and Hagness, 2005; Kunz and Luebbers, 1993). The discrete convolution sums (3) and (4) have been implemented in the FDTD time-stepping process. Hence, the multipole amplitudes $a_{n, m}$ and $b_{n, m}$ are calculated as "on the fly" with the FDTD nearfield solver. During the post-processing stage, the frequency-domain multipole amplitudes have been computed by the means of equations (5) and (6) through a numerical (discrete) Fourier transformation.

The implementation has been applied to determine the radiation pattern of an ultra wide-band balanced antipodal Vivaldi antenna ${ }^{1}$. The antenna is comprised of three perfectly electrically conducting (PEC) layers where the outer ground plane layers are flared in the opposite direction of the $\mathrm{Vi}$ valdi. The substrate of the antenna is $49 \times 90 \times 3.15 \mathrm{~mm}^{3}$ and is constructed of a Duroid material with a relative permittivity of 2.32; see Fig. 1 for a CAD drawing of the structure. In order to obtain a wide-band and well defined spectrum, the antenna feed is driven by a Gaussian modulated sine pulse

$s(t)=E_{0} e^{-\alpha\left(t-\beta \Delta_{t}\right)^{2}} \sin \left(2 \pi f_{c}\left(t-\beta \Delta_{t}\right)\right)$,

with center frequency $f_{c}=5 \mathrm{GHz} . \Delta_{t}=2.9345 \mathrm{ps}$ denotes the temporal increment (in seconds) given by the FDTD solver, the pulse width $\beta$ (in time-steps) is defined by $\beta=\frac{4}{\sqrt{\alpha} \Delta_{t}}=200$, and the loss factor reads $\alpha=\left(\frac{4}{\beta \Delta_{t}}\right)^{2}$. The corresponding frequency spectrum is shown in Fig. 2. The feed is applied in line with the center conductor at the base

\footnotetext{
${ }^{1}$ http://www.remcom.com/xfdtd/examples/ vivaldi-antenna-geometry.html
} 


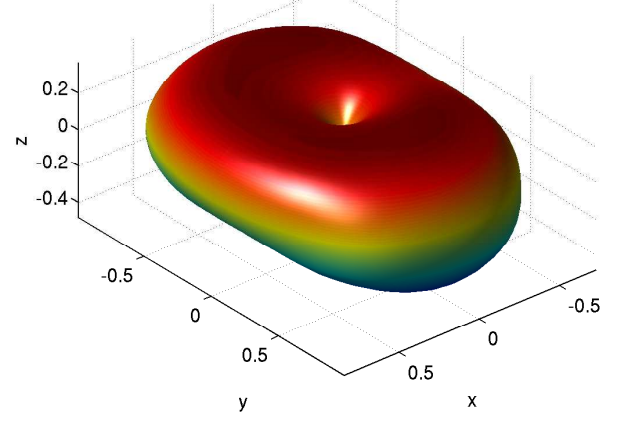

Fig. 3. Three dimensional radiated power pattern (normalized) at $1 \mathrm{GHz}$.

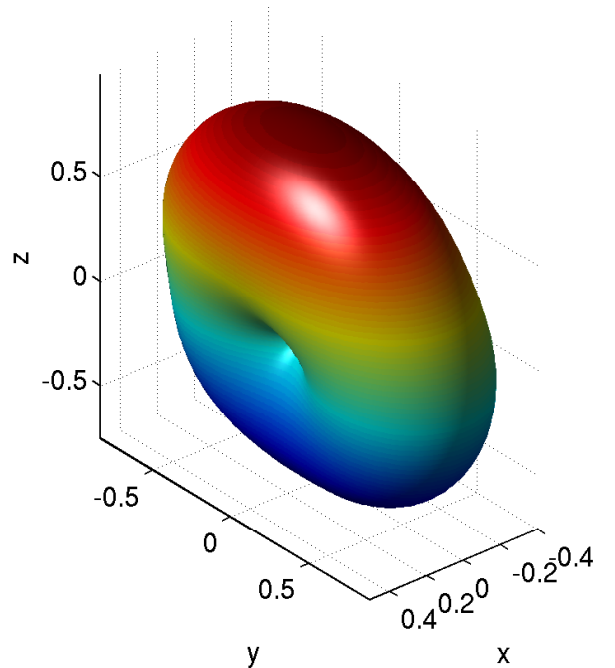

Fig. 4. Three dimensional radiated power pattern (normalized) at $2 \mathrm{GHz}$.

of the strip line. The source resistance at the feed is set to $50 \mathrm{Ohms}$ to best match the input impedance of the antenna. The XFdtd antenna is simulated using $1.5 \times 1.575 \times 1.5 \mathrm{~mm}^{3}$ cells ( $\hat{\boldsymbol{y}}$ dimension modified to match the $3.15 \mathrm{~mm}$ thickness) in a space of $68 \times 43 \times 101$ cells (plus eight cells of PML in each direction). The obtained frequency-domain multipole amplitudes are employed to determine high-resolution threedimensional power radiation patterns of the provided antenna within the entire (discrete) spectrum of the input pulse, which is easily seen to be

$$
\begin{aligned}
P(\omega, \vartheta, \varphi)=\frac{4 \pi}{\kappa_{0}^{2} E_{0}^{2}} \mid \sum_{n, m} j^{n}\left[-A_{n, m}(\omega) \boldsymbol{n}_{n, m}(\vartheta, \varphi)\right. & \\
& \left.\left.\left.-Z_{0} B_{n, m}(\omega) \boldsymbol{m}_{n, m} \vartheta, \varphi\right)\right)\right]\left.\right|^{2}
\end{aligned}
$$

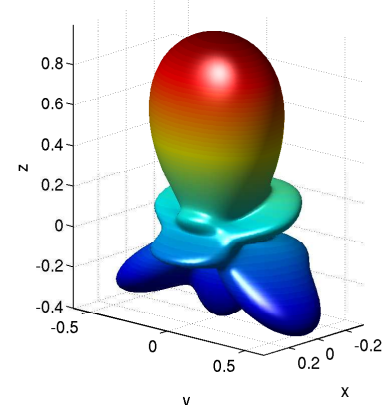

Fig. 5. Three dimensional radiated power pattern (normalized) at $5 \mathrm{GHz}$.

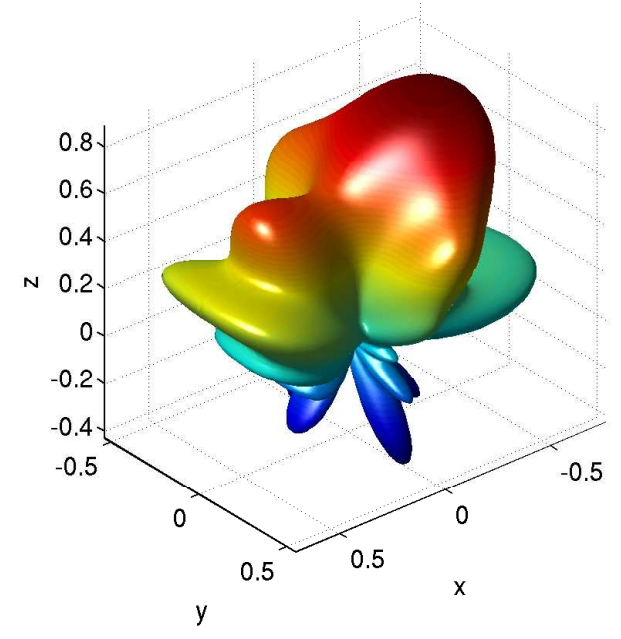

Fig. 6. Three dimensional radiated power pattern (normalized) at $9 \mathrm{GHz}$.

The low computational cost of this post-processing even admits to produce radiation-pattern-films within a few minutes. Figs. 3-6 show the radiated power patterns for an input frequency of $1 \mathrm{GHz}, 2 \mathrm{GHz}, 5 \mathrm{GHz}$ and $9 \mathrm{GHz}$, respectively.

\section{Conclusions}

We have presented an efficient method to calculate frequency-domain near-and far-fields of antennas for a wide frequency spectrum by a single time-domain run of an arbitrary near-field solver. We have demonstrated the applicability of the present method by the implementation into a standard FDTD code including an analysis of an ultra wide-band balanced antipodal Vivaldi antenna. 
Acknowledgements. This work has been supported by the Deutsche Forschungsgemeinschaft.

\section{References}

Abramowitz, M. and Stegun, I. E.: Handbook of Mathematical Functions, New York: Dover Pub., 10 edn., 1972.

Adam, J. and Klinkenbusch, L.: Argument-Recursive Computation of Legendre Polynomials with Applications in Computational Electromagnetics, in: Proc. of the IEEE Antennas and Propagation Society International Symposium with USNC/URSI National Radio Science and AMEREM Meetings, Albuquerque, New Mexico, 2006.

Balanis, C. A.: Advanced Engineering Electromagnetics, Wiley, New York, 1989.

Chu, L.: Physical Limitations of Omni-Directional Antennas, J. App. Physics, 19, 1163-1175, 1948.

Fante, R.: Quality Factor of General Ideal Antennas, IEEE Trans. Antennas Propagat., 17, 151-155, 1969.

Hansen, J.: Spherical Near-Field Antenna Measurements, London: Peter Peregrinus Ltd., 1988.

Remcom Inc.: XFDTD (R) Vers. 4.06, state College, PA 16805 USA, 1998.

Klinkenbusch, L.: A Spherical Multipole Interface for Numerical Methods in Electromagnetic Field Theory, in: Proceedings of the Latsis Symposium on Computational Electromagnetics, 242247, Zürich, Switzerland, 1995.
Klinkenbusch, L. and Oetting, C.-C.: Correction to: Near-to-FarField Transformation by a Time-Domain Spherical-Multipole Analysis, IEEE Trans. on Antennas and Propagation, 56, 3367, 2007.

Kunz, K. and Luebbers, R.: The Finite Difference Time Domain Method for Electromagnetics, Boca Raton: CRC Press, 1993.

Luebbers, R., Kunz, K., Schneider, M., and Hundsberger, F.: A Finite-Difference Time-Domain Near Zone to Far Zone Transformation, IEEE Trans. on Antennas and Propagation, 39, 429433, 1991.

Oetting, C.-C. and Klinkenbusch, L.: Near-to-Far-Field Transformation by a Time-Domain Spherical-Multipole Analysis, IEEE Trans. on Antennas and Propagation, 53, 2054-2063, 2005.

Boag, A. and Shlivinski, A.: Fast Evaluation of the Radiation Patterns of True Time Delay Arrays With Beam Steering, IEEE Transactions on Antennas and Propagation, 55, 3421-3432, 2007.

Taflove, A. and Hagness, S. C.: Computational Electromagnetics: The Finite-Difference Time-Domain Method, Artech House, 3 edn., 2005.

Yaghjian, A. and Best, S.: Impedance, Bandwidth, and $Q$ of Antennas, IEEE Trans. Antennas Propagat., 53, 1298-1324, 2005.

Zhang, S. and Jin, J.: Computation of Special Functions, New York: Wiley, 1996. 